PROCEEDINGS OF THE

AMERICAN MATHEMATICAL SOCIETY

Volume 138, Number 3, March 2010, Pages 1011-1021

S 0002-9939(09)10127-2

Article electronically published on October 22, 2009

\title{
RATES OF CONVERGENCE FOR THE ITERATES OF CESÀRO OPERATORS
}

\author{
JOSÉ A. ADELL AND A. LEKUONA \\ (Communicated by Walter Van Assche)
}

\begin{abstract}
We obtain sharp rates of convergence in the usual sup-norm for the $n$th iterates $D^{n} f$ and $C^{n} f$ of continuous and discrete Cesàro operators, respectively. In both cases the best possible rate of convergence is $n^{-1 / 2}$, and such a rate is attained under appropriate integrability conditions on $f$. Otherwise, the rates of convergence could be extremely poor, depending on the behavior of $f$ near the boundary. We introduce probabilistic representations of $D^{n} f$ and $C^{n} f$ involving standardized sums of independent identically distributed random variables and binomial mixtures, respectively, which allow us to use the classical Berry-Esseen theorem.
\end{abstract}

\section{Introduction AND MAIN RESUlts}

The starting point of this work is a recent paper by Galaz and Solís [6], in which these authors give necessary and sufficient conditions for the convergence in the usual sup-norm of the iterates of continuous and discrete Cesàro operators. Once such characterization criteria have been obtained, the following natural question is to ask for rates of convergence.

Let us recall the results in [6] in which we are interested. Let $C[0, \infty]$ be the set of real continuous functions defined on $[0, \infty)$ such that $\lim _{x \rightarrow \infty} f(x)$ exists. Denote by $D: C[0, \infty] \rightarrow C[0, \infty]$ the continuous Cesàro operator defined by

$$
D f(x)=\frac{1}{x} \int_{0}^{x} f(u) d u, \quad x>0 \quad(D f(0)=f(0)),
$$

and by $D^{n}$ the $n$th iterate of $D$. For a given $f \in C[0, \infty]$, Galaz and Solís [6, Theorem 4] show that the sequence $\left(D^{n} f\right)_{n \geq 1}$ converges in the usual sup-norm if and only if

$$
\lim _{x \rightarrow \infty} f(x)=f(0) .
$$

In such a case, the limit of $D^{n} f$, as $n \rightarrow \infty$, is the constant function $f(0)$. Similarly, denote by $\mathbb{Z}_{+}$the set of non-negative integers and by $\mathbb{N}=\mathbb{Z}_{+} \backslash\{0\}$ the set of natural

Received by the editors March 2, 2009, and, in revised form, July 16, 2009.

2000 Mathematics Subject Classification. Primary 47B37, 60F05.

Key words and phrases. Cesàro operator, iterates, rate of convergence, Berry-Esseen theorem, binomial mixture.

This work has been supported by research grants MTM2008-06281-C02-01/MTM and DGA E-64 and by FEDER funds.

(C)2009 American Mathematical Society Reverts to public domain 28 years from publication 
numbers. Let $c$ be the set of real functions defined on $\mathbb{Z}_{+}$such that $\lim _{m \rightarrow \infty} f(m)$ exists, and denote by $C: c \rightarrow c$ the discrete Cesàro operator defined as

$$
C f(m)=\frac{f(0)+\ldots+f(m)}{m+1}, \quad m \in \mathbb{Z}_{+} .
$$

It turns out (cf. [6, Theorem 1]) that, given $f \in c$, the sequence $\left(C^{n} f\right)_{n \geq 1}$ converges if and only if

$$
\lim _{m \rightarrow \infty} f(m)=f(0)
$$

and, if so, the limit of $C^{n} f$, as $n \rightarrow \infty$, is the constant function $f(0)$.

In any of the previous results, no generality is lost if we assume that $f(0)=0$ since, otherwise, we can replace $f$ by the translated function $f-f(0)$. On the other hand, it will be shown in Theorems 1.1 and 1.2 below that the continuity of $f$ in $(0, \infty)$ is not essential to guarantee the convergence of $\left(D^{n} f\right)_{n \geq 1}$. For these reasons, we slightly enlarge the set of functions on which the continuous Cesàro operator acts. In fact, denote by $\mathcal{B}[0, \infty]$ the set of bounded measurable real functions $f$ defined on $[0, \infty)$ such that

$$
\lim _{x \rightarrow \infty} f(x)=\lim _{x \rightarrow 0} f(x)=f(0)=0 .
$$

In the same way, denote by $\mathcal{B}\left(\mathbb{Z}_{+}\right)$the set of real functions $f$ defined on $\mathbb{Z}_{+}$such that

$$
\lim _{m \rightarrow \infty} f(m)=f(0)=0 .
$$

Observe that these sets are preserved by the corresponding Cesàro operators, i.e., $D: \mathcal{B}[0, \infty] \rightarrow \mathcal{B}[0, \infty]$ and $C: \mathcal{B}\left(\mathbb{Z}_{+}\right) \rightarrow \mathcal{B}\left(\mathbb{Z}_{+}\right)$.

To state our main results, the following notation will be needed. Let $\mathcal{S}(\mathbb{R})$ be the set of symmetric real functions $g$ defined on $\mathbb{R}$, such that $g$ is non-increasing on $[0, \infty)$ and $\lim _{x \rightarrow \infty} g(x)=0$. We define the symmetrization operator $M$ : $\mathcal{B}[0, \infty] \rightarrow \mathcal{S}(\mathbb{R})$ as

$$
M f(x)=\sup _{|u| \geq|x|}\left\{|f|\left(e^{-u}\right),|f|\left(e^{u}\right)\right\}, \quad x \in \mathbb{R}, \quad f \in \mathcal{B}[0, \infty] .
$$

The sup-norm and the $L^{1}$-norm of a given function $f$ are denoted by $\|f\|_{\infty}$ and $\|f\|_{1}$, respectively. Finally, if $\left(x_{n}\right)_{n \geq 1}$ and $\left(y_{n}\right)_{n \geq 1}$ are two sequences of positive real numbers, we write $x_{n} \approx y_{n}$ whenever

$$
a=\varliminf_{n \rightarrow \infty} \frac{x_{n}}{y_{n}} \leq \varlimsup_{n \rightarrow \infty} \frac{x_{n}}{y_{n}}=b,
$$

for some $0<a \leq b<\infty$, whereas we write $x_{n} \gtrsim y_{n}$ if (1.4) holds for some $0<a \leq b \leq \infty$. We are in a position to state the following.

Theorem 1.1. Let $f \in \mathcal{B}[0, \infty]$ and $n \in \mathbb{N}$. For any $a>0$, we have

$$
\left\|D^{n} f\right\|_{\infty} \leq \frac{2 a}{\sqrt{2 \pi}} \operatorname{DMf}(a \sqrt{n})+2 M f(a \sqrt{n})+\frac{2.8224(6-e)\|f\|_{\infty}}{e \sqrt{n}} .
$$

If, in addition, $M f$ is integrable, then

$$
\left\|D^{n} f\right\|_{\infty} \leq\left(\frac{\|M f\|_{1}}{\sqrt{2 \pi}}+\frac{2.8224(6-e)\|f\|_{\infty}}{e}\right) \frac{1}{\sqrt{n}} .
$$


To discuss the rates of convergence in Theorem 1.1 set $a=1$. Since $M f$ is non-increasing on $[0, \infty)$, we see from (1.1) that $\operatorname{DMf}(\sqrt{n}) \geq M f(\sqrt{n}), n \in \mathbb{N}$. Again by (1.1), $D M f(\sqrt{n}) \gtrsim n^{-1 / 2}$, and $D M f(\sqrt{n}) \approx n^{-1 / 2}$ if and only if $M f$ is integrable.

To give meaning to the $L^{1}$-norm $\|M f\|_{1}$ on the right-hand side in (1.6), let us start with an integrable function $g \in \mathcal{S}(\mathbb{R})$. Then, the function $f(x)=g(\log x)$, $x \geq 0$, belongs to $\mathcal{B}[0, \infty]$ and satisfies $M f=g$. We therefore have in this case

$$
\|M f\|_{1}=\|g\|_{1}=\int_{0}^{\infty} \frac{f(x)}{x} d x .
$$

In the following result, we show that the rates of convergence in (1.5) and (1.6) are accurate. In this respect, denote by $\phi$ the standard normal probability density, i.e.,

$$
\phi(x)=\frac{1}{\sqrt{2 \pi}} e^{-x^{2} / 2}, \quad x \in \mathbb{R} .
$$

Theorem 1.2. Let $g \in \mathcal{S}(\mathbb{R})$ and $n \in \mathbb{N}$. Let $f \in \mathcal{B}[0, \infty]$ be defined as $f(x)=$ $g(\log x), x \geq 0$. Then,

(a) If $g$ is not integrable, we have for any $a>0$

$$
\begin{aligned}
& 2 a \phi(a) D g(a \sqrt{n})-\frac{2.8224(6-e)\|g\|_{\infty}}{e \sqrt{n}} \leq\left\|D^{n} f\right\|_{\infty} \\
& \leq \frac{2 a}{\sqrt{2 \pi}} D g(a \sqrt{n})+2 g(a \sqrt{n})+\frac{2.8224(6-e)\|g\|_{\infty}}{e \sqrt{n}} .
\end{aligned}
$$

(b) If $g$ is integrable, then

$$
\frac{C_{n}}{\sqrt{n}} \int_{-\sqrt{n}}^{\sqrt{n}} g(u) d u \leq\left\|D^{n} f\right\|_{\infty} \leq\left(\frac{\|g\|_{1}}{\sqrt{2 \pi}}+\frac{2.8224(6-e)\|g\|_{\infty}}{e}\right) \frac{1}{\sqrt{n}},
$$

where the positive sequence $\left(C_{n}\right)_{n \geq 1}$ converges to $1 / \sqrt{2 \pi e}$, as $n \rightarrow \infty$.

In essence, Theorem 1.2 says that the rate of convergence of $\left\|D^{n} f\right\|_{\infty}$ is $n^{-1 / 2}$ or $D g(\sqrt{n})$, according to whether $g$ is integrable or not. Both Theorems 1.1 and 1.2 allow us to conclude the following. Generally speaking, the best possible rate of convergence of the iterates $D^{n} f$ is $n^{-1 / 2}$. Such a rate is attained if $M f$ is integrable. Otherwise, the rate of convergence of $D^{n} f$ could be extremely poor, depending on the behavior of $M f$ at infinity, as follows from (1.8).

Theorem 1.1 can also be applied to the iterates $C^{n}$ of the discrete Cesàro operator, after making use of the Szàsz operator $L: \mathcal{B}\left(\mathbb{Z}_{+}\right) \rightarrow \mathcal{B}[0, \infty]$ defined by

$$
L f(x)=\sum_{k=0}^{\infty} f(k) \frac{e^{-x} x^{k}}{k !}, \quad x \geq 0, \quad f \in \mathcal{B}\left(\mathbb{Z}_{+}\right) .
$$

Actually, we state the following.

Theorem 1.3. Let $f \in \mathcal{B}\left(\mathbb{Z}_{+}\right)$and $n \in \mathbb{N}$. For any $a>0$, we have

$$
\begin{aligned}
\left\|C^{n} f\right\|_{\infty} \leq & \frac{2 a}{\sqrt{2 \pi}} \operatorname{DMLf}(a \sqrt{n})+2 M L f(a \sqrt{n}) \\
& +\frac{2.8224(6-e)\|f\|_{\infty}}{e \sqrt{n}}+\frac{\|f\|_{\infty}}{2^{n-1}} .
\end{aligned}
$$


If, in addition, $M L f$ is integrable, then

$$
\left\|C^{n} f\right\|_{\infty} \leq\left(\frac{\|M L f\|_{1}}{\sqrt{2 \pi}}+\frac{2.8224(6-e)\|f\|_{\infty}}{e}\right) \frac{1}{\sqrt{n}}+\frac{\|f\|_{\infty}}{2^{n-1}} .
$$

Although we omit the details, analogous statements to those contained in Theorem 1.2 and the comments following it can be made with regard to the iterates $C^{n}$. Despite these facts, Fridy et al. show in [5. Theorem 3.4] that, in the context of the central limit theorem, the classical Cesàro summability method gives the best possible rates of convergence among all regular triangular summability methods with rows adding up to one.

Let us say something about the proofs. The main point to prove Theorems 1.1 and 1.2 is a probabilistic representation of the iterates $D^{n} f$ in terms of a standardized sum of independent identically distributed random variables having the exponential distribution with unit mean (see formula (2.8)). After developing some elementary techniques concerning translation operators built up from symmetric random variables (see Section 3), such a probabilistic representation allows us to use the well known Berry-Esseen theorem. This explains why the best possible rate of convergence of $\left\|D^{n} f\right\|_{\infty}$ is $n^{-1 / 2}$, which coincides with the speed of convergence in the classical version of the central limit theorem. The constant 2.8224 in Theorems 1.1 and 1.2 comes from the refinement of the Berry-Esseen theorem recently obtained by Shevtshova 8 .

Concerning Theorem 1.3, the key point is the probabilistic representation of $C^{n}$ in terms of a binomial mixture whose mixing distribution is a product of $n$ independent identically distributed random variables having the uniform distribution on $[0,1]$ (see formula (2.6) ). By approximating this binomial mixture to an appropriate Poisson mixture and using the well known estimates for the total variation distance between binomial and Poisson distributions having the same mean, we can derive the results for $C^{n}$ from those obtained for $D^{n}$.

Finally, it should be observed that the probabilistic representations for both $D^{n}$ and $C^{n}$ provide an independent proof of the analytical expressions of these iterates already shown by Hardy [7] and Boyd [4, respectively (see the comments after Proposition 2.3).

\section{Probabilistic Representations}

From now on, every random variable $X$ is assumed to be real-valued. If $F(x)=$ $P(X \leq x), x \in \mathbb{R}$, is the distribution function of $X$ and $f$ is a bounded measurable real function defined on $\mathbb{R}$, the expectation of $f(X)$ is denoted by

$$
E f(X)=\int_{\mathbb{R}} f(x) d F(x) .
$$

The symbol $X=(\mathcal{L}) Y$ means that the random variables $X$ and $Y$ have the same distribution functions. Finally, all of the random variables appearing in the same expression are supposed to be mutually independent.

Let $\left(V_{k}\right)_{k \geq 1}$ be a sequence of independent identically distributed random variables having the uniform distribution on $[0,1]$. Denote by $1_{A}$ the indicator function of the set $A$. For each $m \in \mathbb{N}$, the random variable

$$
S_{m}(t)=\sum_{k=1}^{m} 1_{[0, t]}\left(V_{k}\right), \quad 0 \leq t \leq 1 \quad\left(S_{0}(t) \equiv 0\right),
$$


has the binomial distribution with parameters $m$ and $t$, that is,

$$
P\left(S_{m}(t)=k\right)=\left(\begin{array}{c}
m \\
k
\end{array}\right) t^{k}(1-t)^{m-k}, \quad k=0,1, \ldots, m .
$$

Let $T$ be a random variable taking values in $[0,1]$ with distribution function $F$. Assume that $T$ is independent of the sequence $\left(V_{k}\right)_{k \geq 1}$. For each $m \in \mathbb{N}$, the random variable $S_{m}(T)$ is called a binomial mixture with mixing random variable $T$. The probability law of $S_{m}(T)$ is given by

$$
\begin{aligned}
P\left(S_{m}(T)=k\right) & =\int_{0}^{1} P\left(S_{m}(t)=k\right) d F(t) \\
& =\left(\begin{array}{c}
m \\
k
\end{array}\right) \int_{0}^{1} t^{k}(1-t)^{m-k} d F(t), \quad k=0,1, \ldots, m .
\end{aligned}
$$

In particular, if $T$ has the uniform distribution on $[0,1]$, then

$$
P\left(S_{m}(T)=k\right)=\left(\begin{array}{c}
m \\
k
\end{array}\right) \int_{0}^{1} t^{k}(1-t)^{m-k} d t=\frac{1}{m+1}, \quad k=0,1, \ldots, m .
$$

As follows from (2.1), the characteristic function of $S_{m}(T)$ is given by

$$
\begin{aligned}
E e^{i \lambda S_{m}(T)} & =\int_{0}^{1} E e^{i \lambda S_{m}(t)} d F(t)=\int_{0}^{1}\left(\left(e^{i \lambda}-1\right) t+1\right)^{m} d F(t) \\
& =E\left(\left(e^{i \lambda}-1\right) T+1\right)^{m}, \quad \lambda \in \mathbb{R} .
\end{aligned}
$$

To obtain a probabilistic representation of the iterates of Cesàro operators, the following two auxiliary results will be needed. In this respect, let $\left(\tilde{V}_{k}\right)_{k \geq 1}$ be an independent copy of $\left(V_{k}\right)_{k \geq 1}$ and set

$$
\tilde{S}_{m}(t)=\sum_{k=1}^{m} 1_{[0, t]}\left(\tilde{V}_{k}\right), \quad 0 \leq t \leq 1, \quad m \in \mathbb{N} .
$$

Lemma 2.1. Let $T$ and $R$ be two random variables taking values in $[0,1]$. Then,

$$
S_{\tilde{S}_{m}(R)}(T)=(\mathcal{L}) S_{m}(R T) .
$$

Proof. Let $\lambda \in \mathbb{R}$ and let $G$ be the distribution function of $R$. By (2.3), we have

$$
\begin{aligned}
& E \exp \left(i \lambda S_{\tilde{S}_{m}(R)}(T)\right)=E\left(\left(e^{i \lambda}-1\right) T+1\right)^{\tilde{S}_{m}(R)} \\
& =\int_{0}^{1} E\left(\left(e^{i \lambda}-1\right) T+1\right)^{\tilde{S}_{m}(r)} d G(r)=\int_{0}^{1} E\left(\left(e^{i \lambda}-1\right) T r+1\right)^{m} d G(r) \\
& =E\left(\left(e^{i \lambda}-1\right) T R+1\right)^{m} .
\end{aligned}
$$

The conclusion follows from (2.3), (2.4), and the uniqueness theorem for characteristic functions.

Although the following result is a particular case of [2, Lemma 1], we will give here a simple proof of it which will be useful for our purposes.

Lemma 2.2. Let $\left(U_{k}\right)_{k \geq 1}$ be a sequence of independent identically distributed random variables having the uniform distribution on $[0,1]$. For any $n \in \mathbb{N}$, the random variable $Y_{n}=U_{1} \cdots U_{n}$ has probability density

$$
\rho_{n}(x)=\frac{1}{(n-1) !}\left(\log \frac{1}{x}\right)^{n-1}, \quad 0<x \leq 1 .
$$


Proof. Let $n \in \mathbb{N}$. For each $i=1, \ldots, n$, the random variable $-\log U_{i}$ has the exponential distribution with unit mean. Thus, the random variable $-\log Y_{n}=$ $-\log U_{1}-\ldots-\log U_{n}$ has the gamma density

$$
\psi_{n}(x)=\frac{1}{(n-1) !} x^{n-1} e^{-x}, \quad x \geq 0 .
$$

This readily implies that the probability density of $Y_{n}$ is that defined in (2.5).

Proposition 2.3. Let $n \in \mathbb{N}$ and let $\left(U_{k}\right)_{k \geq 1}$ be a sequence of independent identically distributed random variables having the uniform distribution on $[0,1]$. Set $Y_{n}=U_{1} \cdots U_{n}$. Then:

(a) For any $f \in \mathcal{B}\left(\mathbb{Z}_{+}\right)$and $m \in \mathbb{Z}_{+}$, we have

$$
\begin{aligned}
C^{n} f(m) & =\operatorname{Ef}\left(S_{m}\left(Y_{n}\right)\right) \\
& =\frac{1}{(n-1) !} \sum_{k=0}^{m} f(k)\left(\begin{array}{c}
m \\
k
\end{array}\right) \int_{0}^{1} t^{k}(1-t)^{m-k}\left(\log \frac{1}{t}\right)^{n-1} d t .
\end{aligned}
$$

(b) For any $f \in \mathcal{B}[0, \infty]$ and $x \geq 0$, we have

$$
D^{n} f(x)=E f\left(x Y_{n}\right)=\frac{1}{(n-1) !} \int_{0}^{1} f(x t)\left(\log \frac{1}{t}\right)^{n-1} d t,
$$

as well as

$$
D^{n} f(x)=E f\left(e^{-\left(\sqrt{n} S_{n}+n-\log x\right)}\right),
$$

where $S_{n}$ is the standardized sum

$$
S_{n}=-\frac{1}{\sqrt{n}} \sum_{i=1}^{n}\left(\log U_{i}+1\right) .
$$

Proof. We shall use induction as follows. The formula

$$
C^{1} f(m)=E f\left(S_{m}\left(U_{1}\right)\right)
$$

is an immediate consequence of (1.2) and (2.2). Also,

$$
C^{n+1} f(m)=E C^{1} f\left(S_{m}\left(Y_{n}\right)\right)=E f\left(S_{\tilde{S}_{m}\left(U_{n+1}\right)}\left(Y_{n}\right)\right)=E f\left(S_{m}\left(Y_{n+1}\right)\right),
$$

as follows from (2.10) and Lemma 2.1 This, in conjunction with (2.5), shows (2.6).

On the other hand, it is clear from (1.1) that

$$
D^{1} f(x)=E f\left(x U_{1}\right),
$$

thus implying that

$$
D^{n+1} f(x)=E D^{1} f\left(x Y_{n}\right)=E f\left(x Y_{n+1}\right) .
$$

Therefore, formula (2.7) follows from (2.5). Finally, formula (2.8) is just a reformulation of the first equality in (2.7). The proof is complete.

It should be mentioned that the analytical expressions for $C^{n} f$ and $D^{n} f$ in formulae (2.6) and (2.7) were already found by Hardy [7, Sect. II. 12] and Boyd [4, Lemma 2], respectively. The representation of $D^{n} f$ in terms of the standardized sum $S_{n}$ in formula (2.8) is the crucial point to obtain rates of convergence, since such a representation allows us to use the well known Berry-Esseen bounds for $S_{n}$. This will become apparent in Section 4. 


\section{TRANSLATION OPERATORS AND SYMMETRY}

In this section, we collect some of the tools needed to prove our main results, mainly concerned with translation operators and symmetric random variables. To this end, let $\mathcal{B}$ be the set of bounded measurable functions $g: \mathbb{R} \rightarrow \mathbb{R}$, and let $Y$ be a random variable with distribution function $F$. We consider the translation operator $T: \mathcal{B} \rightarrow \mathcal{B}$ defined as

$$
T g(x)=E g(Y+x), \quad x \in \mathbb{R}, \quad g \in \mathcal{B} .
$$

Recall that the random variable $Y$ is said to be continuous if $F$ is as well and symmetric if $F(y)=P(Y \leq y)=P(-Y \leq y), y \in \mathbb{R}$.

Lemma 3.1. Let $\tilde{g} \in \mathcal{B}$ be a right-continuous function of bounded variation such that $\tilde{g}(x) \rightarrow 0$, as $x \rightarrow-\infty$. If $Y$ is continuous and $g \in \mathcal{B}$ differs from $\tilde{g}$ in a countable set at most, then $T g$ is continuous.

Proof. Let $x \in \mathbb{R}$. $Y$ being continuous, we have from Fubini's theorem

$$
T g(x)=E \tilde{g}(Y+x)=E \int_{(-\infty, Y+x]} d \tilde{g}(y)=\int_{\mathbb{R}}(1-F(y-x)) d \tilde{g}(y) .
$$

Therefore, by the dominated convergence theorem, the continuity of $F$ implies the continuity of $T g$.

Proposition 3.2. Suppose that the random variable $Y$ is continuous a nd symmetric. Then the following are equivalent:

(a) $T g \in \mathcal{S}(\mathbb{R})$, for any $g \in \mathcal{S}(\mathbb{R})$.

(b) $T 1_{[-a, a]} \in \mathcal{S}(\mathbb{R})$, for any $a \geq 0$.

(c) For any $\delta \geq 0$, the function $F_{\delta}:[0, \infty) \rightarrow[0, \infty)$ defined by

$$
F_{\delta}(x)=F(x+\delta)-F(x), \quad x \geq 0,
$$

is non-increasing.

In such a case, we have for any $g \in \mathcal{S}(\mathbb{R})$

$$
\sup _{x \in \mathbb{R}} T g(x)=T g(0)=E g(Y) .
$$

Proof. (b) $\Rightarrow(\mathrm{c})$. Let $a \geq 0$. Since $Y$ is continuous and symmetric, we have

$$
T 1_{[-a, a]}(x)=E 1_{[-a, a]}(-Y+x)=F(x+a)-F(x-a), \quad x \in \mathbb{R} .
$$

This implies (c), because $T 1_{[-a, a]}$ is non-increasing in $[a, \infty)$.

(c) $\Rightarrow($ a). Assume first that $g$ is a continuous function in $\mathcal{S}(\mathbb{R})$. Due to the continuity and symmetry of $g$, it can be verified using standard methods that

$$
\int_{-\infty}^{0} f(y) d g(y)=-\int_{0}^{\infty} f(-y) d g(y)
$$

for any bounded measurable function $f$ defined on $(-\infty, 0]$. Therefore, using the symmetry of $Y$, we have as in (3.1)

$$
T g(x)=\int_{\mathbb{R}} F(x-y) d g(y)=-\int_{0}^{\infty}(F(x+y)-F(x-y)) d g(y), \quad x \in \mathbb{R} .
$$

This, together with (3.2), shows that $T g \in \mathcal{S}(\mathbb{R})$. 
Suppose now that $g \in \mathcal{S}(\mathbb{R})$ is arbitrary. Let $V$ be a random variable independent of $Y$ having the uniform distribution on $[-1,1]$. For any $h>0$, we consider the function

$$
g_{h}(x)=E g(h V+x)=\frac{1}{2 h} \int_{x-h}^{x+h} g(u) d u, \quad x \in \mathbb{R},
$$

as well as the translation operator

$$
T_{h} g(x)=E g(Y+h V+x)=E T g(h V+x)=T g_{h}(x), \quad x \in \mathbb{R} .
$$

It can be checked that $g_{h}$ is a continuous function in $\mathcal{S}(\mathbb{R})$. By (3.4) and the first part of this proof, we see that $T g_{h} \in \mathcal{S}(\mathbb{R})$. Again by (3.4) and the dominated convergence theorem, we obtain $T_{h} g(x) \rightarrow T g(x), x \in \mathbb{R}$, as $h \rightarrow 0$, since $T g$ is continuous by virtue of Lemma 3.1. This implies that $T g \in \mathcal{S}(\mathbb{R})$.

Finally, implication $(\mathrm{a}) \Rightarrow(\mathrm{b})$ is obvious, and (3.3) is an immediate consequence of statement (a). The proof is complete.

Remark 3.3. If the random variable $Y$ is absolutely continuous with probability density $\rho \in \mathcal{S}(R)$, then assertion (c) in Proposition 3.2 trivially holds, in particular, if $Y=(\mathcal{L}) Z$, where $Z$ is a standard normal random variable with probability density $\phi$, as defined in (1.7).

Proposition 3.4. Let $Z$ be a standard normal random variable and $g \in \mathcal{S}(\mathbb{R})$. For any $n \in \mathbb{N}$ and $a>0$, we have

$$
a \phi(a) D g(a \sqrt{n}) \leq \frac{1}{2} E g(\sqrt{n} Z) \leq \frac{a}{\sqrt{2 \pi}} D g(a \sqrt{n})+P(Z \geq a) g(a \sqrt{n}) .
$$

As a consequence, $E g(\sqrt{n} Z) \gtrsim n^{-1 / 2}$ and $E g(\sqrt{n} Z) \approx n^{-1 / 2}$ if and only if $g$ is integrable. Under this last assumption, we have

$$
E g(\sqrt{n} Z) \leq \frac{\|g\|_{1}}{\sqrt{2 \pi}} \frac{1}{\sqrt{n}}
$$

Proof. Using (1.1), (1.7), and the fact that $g \in \mathcal{S}(\mathbb{R})$, we have

$$
\begin{aligned}
\frac{1}{2} E g(\sqrt{n} Z) & =\int_{0}^{a} g(\sqrt{n} x) \phi(x) d x+\int_{a}^{\infty} g(\sqrt{n} x) \phi(x) d x \\
& \leq \frac{a}{\sqrt{2 \pi}} D g(a \sqrt{n})+P(Z \geq a) g(a \sqrt{n}) .
\end{aligned}
$$

The remaining assertions are proved in a similar way.

Our last auxiliary result is the following.

Proposition 3.5. Let $\tilde{g} \in \mathcal{B}$ be a right-continuous function of bounded variation such that $\tilde{g}(x) \rightarrow 0$ as $x \rightarrow-\infty$, and let $X$ and $Y$ be continuous random variables. If $g \in \mathcal{B}$ differs from $\tilde{g}$ in a countable set at most, then

$$
|E g(X)-E g(Y)| \leq V(\tilde{g}) \sup _{x \in \mathbb{R}}|P(X \leq x)-P(Y \leq y)|,
$$

where $V(\tilde{g})$ stands for the total variation of $\tilde{g}$. 
Proof. Let $F$ and $G$ be the distribution functions of $X$ and $Y$, respectively. By assumption and Fubini's theorem, we have

$$
\begin{aligned}
E g(X)-E g(Y) & =\int_{\mathbb{R}} d(F-G)(x) \int_{(-\infty, x]} d \tilde{g}(u)=\int_{\mathbb{R}} d \tilde{g}(u) \int_{[u, \infty)} d(F-G)(x) \\
& =\int_{\mathbb{R}}(G(u)-F(u)) d \tilde{g}(u),
\end{aligned}
$$

thus implying (3.7) and completing the proof.

\section{The PROOFS}

Recently, Shevtsova [8] has obtained the following improvement of the constant in the classical Berry-Esseen theorem. Suppose that $\left(X_{n}\right)_{n \geq 1}$ is a sequence of independent identically distributed random variables such that $E X_{1}=0, E X_{1}^{2}=$ $\sigma^{2}>0$, and $E\left|X_{1}\right|^{3}=\beta<\infty$. For any $n \in \mathbb{N}$, consider the standardized sum $\tilde{S}_{n}=\left(X_{1}+\ldots+X_{n}\right) /(\sigma \sqrt{n})$. Then,

$$
\sup _{x \in \mathbb{R}}\left|P\left(\tilde{S}_{n} \leq x\right)-P(Z \leq x)\right| \leq \frac{0.7056 \beta}{\sigma^{3}} \frac{1}{\sqrt{n}},
$$

where $Z$ is a standard normal random variable.

Proof of Theorem 1.1. Let $f \in \mathcal{B}[0, \infty], x \geq 0$, and $n \in \mathbb{N}$. By (1.3) and (2.8), we have

$$
\begin{aligned}
\left|D^{n} f(x)\right| \leq & E|f|\left(e^{-\left(\sqrt{n} S_{n}+n-\log x\right)}\right) \leq \operatorname{EMf}\left(\sqrt{n} S_{n}+n-\log x\right) \\
\leq & \left|E M f\left(\sqrt{n} S_{n}+n-\log x\right)-\operatorname{EMf}(\sqrt{n} Z+n-\log x)\right| \\
& +E M f(\sqrt{n} Z+n-\log x),
\end{aligned}
$$

where $S_{n}$ is the standardized sum defined in (2.9), i.e.,

$$
S_{n}=\frac{1}{\sqrt{n}} \sum_{i=1}^{n}\left(X_{i}-1\right), \quad X_{i}=-\log U_{i}, \quad i=1, \ldots, n .
$$

Since $X_{1}$ has the exponential distribution with unit mean, as seen in the proof of Lemma 2.2, it can be checked that $E\left(X_{1}-1\right)=0, E\left(X_{1}-1\right)^{2}=1$, and $E\left|X_{1}-1\right|^{3}=2(6-e) e^{-1}$. We therefore have from (4.1)

$$
\sup _{x \in \mathbb{R}}\left|P\left(S_{n} \leq x\right)-P(Z \leq x)\right| \leq \frac{1.4112(6-e)}{e \sqrt{n}} .
$$

On the other hand, since $M f \in \mathcal{S}(\mathbb{R}), M f$ differs in a countable set at most from a right-continuous function of bounded variation whose total variation is $2\|M f\|_{\infty}=$ $2\|f\|_{\infty}$, as follows from (1.3). Thus, by Proposition 3.5 and (4.3), the first term on the right-hand side in (4.2) is bounded above by

$$
2\|f\|_{\infty} \sup _{x \in \mathbb{R}}\left|P\left(S_{n} \leq x\right)-P(Z \leq x)\right| \leq \frac{2.8224(6-e)\|f\|_{\infty}}{e \sqrt{n}} .
$$

On the other hand, let $a>0$. From (3.3) and (3.5), we see that

$$
E M f(\sqrt{n} Z+n-\log x) \leq E M f(\sqrt{n} Z) \leq \frac{2 a}{\sqrt{2 \pi}} D M f(a \sqrt{n})+2 M f(a \sqrt{n}) .
$$

Estimate (1.5) follows from (4.4) and (4.5). The proof of estimate (1.6) follows a similar pattern by replacing (3.5) by (3.6). 
Proof of Theorem 1.2 , Let $n \in \mathbb{N}, g \in \mathcal{S}(\mathbb{R})$, and $f(x)=g(\log x), x \geq 0$. As noted in the comments following Theorem 1.1, we have $M f=g$. Therefore, the upper bounds in (1.8) and (1.9) readily follow from (1.5) and (1.6), respectively. On the other hand, we write

$$
\begin{aligned}
& E g(\sqrt{n} Z+n-\log x) \\
& =E g(\sqrt{n} Z+n-\log x)-E g\left(\sqrt{n} S_{n}+n-\log x\right)+E f\left(e^{-\left(\sqrt{n} S_{n}+n-\log x\right)}\right) .
\end{aligned}
$$

Hence, we have from (2.8) and (3.3)

$$
\begin{aligned}
E g(\sqrt{n} Z)= & \sup _{x \geq 0} E g(\sqrt{n} Z+n-\log x) \\
\leq & \sup _{x \geq 0}\left|E g(\sqrt{n} Z+n-\log x)-E g\left(\sqrt{n} S_{n}+n-\log x\right)\right| \\
& +\left\|D^{n} f\right\|_{\infty} .
\end{aligned}
$$

Let $a>0$. Using the lower bound in (3.5) and the same argument to show (4.4), we obtain from (4.6)

$$
2 a \phi(a) D g(a \sqrt{n}) \leq \frac{2.8224(6-e)\|g\|_{\infty}}{e \sqrt{n}}+\left\|D^{n} f\right\|_{\infty},
$$

thus showing the lower bound in (1.8). Finally, denote by $\phi_{n}$ the probability density of the random variable $S_{n}$ and by $C_{n}=\min \left\{\phi_{n}(x):-1 \leq x \leq 1\right\}$. We have from (2.8)

$$
\begin{aligned}
\left\|D^{n} f\right\|_{\infty} & =\sup _{x \geq 0} E g\left(\sqrt{n} S_{n}+n-\log x\right) \geq E g\left(\sqrt{n} S_{n}\right) \\
& \geq \int_{-1}^{1} g(\sqrt{n} x) \phi_{n}(x) d x \geq \frac{C_{n}}{\sqrt{n}} \int_{-\sqrt{n}}^{\sqrt{n}} g(u) d u .
\end{aligned}
$$

By the local limit theorem, or simply by calculus, we have

$$
\lim _{n \rightarrow \infty} C_{n}=\min _{-1 \leq x \leq 1} \phi(x)=\frac{1}{\sqrt{2 \pi e}} .
$$

This shows the lower bound in (1.9) and completes the proof.

Let $\left(N_{x}\right)_{x \geq 0}$ be a standard Poisson process, i.e., a stochastic process with independent stationary increments starting at 0 and such that, for any $x>0$, the random variable $N_{x}$ has the Poisson distribution with mean $x$, that is,

$$
P\left(N_{x}=k\right)=\frac{e^{-x} x^{k}}{k !}, \quad k \in \mathbb{Z}_{+} .
$$

Observe that the Szàsz operator $L$ defined in (1.10) can be written as

$$
L f(x)=E f\left(N_{x}\right), \quad x \geq 0, \quad f \in \mathcal{B}\left(\mathbb{Z}_{+}\right) .
$$

On the other hand, recall that the total variation distance between two $\mathbb{Z}_{+}$-valued random variables $X$ and $Y$ is defined by

$$
d_{T V}(X, Y)=\frac{1}{2} \sum_{k=0}^{\infty}|P(X=k)-P(Y=k)| .
$$

Estimates of the total variation distance between binomial and Poisson distributions with the same mean are well known (see Adell et al. [1] and the references therein). 
Let $m \in \mathbb{Z}_{+}$and $0 \leq t \leq 1$. The following estimate in the monograph by Barbour et al. [3, p. 34] will be suitable for our purposes:

$$
d_{T V}\left(S_{m}(t), N_{m t}\right) \leq t .
$$

For any $f \in \mathcal{B}\left(\mathbb{Z}_{+}\right)$, this implies that

$$
\left|E f\left(S_{m}(t)\right)-E f\left(N_{m t}\right)\right| \leq 2\|f\|_{\infty} d_{T V}\left(S_{m}(t), N_{m t}\right) \leq 2\|f\|_{\infty} t .
$$

Proof of Theorem 1.3. Let $f \in \mathcal{B}\left(\mathbb{Z}_{+}\right), n \in \mathbb{N}$, and $m \in \mathbb{Z}_{+}$. From (2.6) we have

$$
\left|C^{n} f(m)\right| \leq\left|E f\left(S_{m}\left(Y_{n}\right)\right)-E f\left(N_{m Y_{n}}\right)\right|+\left|E f\left(N_{m Y_{n}}\right)\right| .
$$

By (4.8), the first term on the right-hand side in (4.9) is bounded above by

$$
2\|f\|_{\infty} E Y_{n}=\frac{\|f\|_{\infty}}{2^{n-1}} .
$$

By virtue of (2.7) and (4.7), we can write

$$
E f\left(N_{m Y_{n}}\right)=E L f\left(m Y_{n}\right)=D^{n} L f(m) .
$$

Note that $L f \in \mathcal{B}[0, \infty]$ and that $\|L f\|_{\infty} \leq\|f\|_{\infty}$, as follows from (4.7). We can therefore apply Theorem 1.1 to bound the term in (4.11). Thus, the conclusion follows from (4.9)-(4.11) and Theorem 1.1

\section{ACKNOWLEDGEMENT}

We thank the referee for a careful reading of the manuscript and helpful comments.

\section{REFERENCES}

[1] J.A. Adell, J.M. Anoz, and A. Lekuona, Exact values and sharp estimates for the total variation distance between binomial and Poisson distributions, Adv. in Appl. Prob. 40, no. 4 (2008), 1033-1047. MR2488531

[2] J.A. Adell, J. de la Cal, and A. Pérez-Palomares, On the Cheney and Sharma operator, J. Math. Anal. Appl. 200 (1996), 663-679. MR.1393107 (97g:41015)

[3] A.D. Barbour, L. Holst, and S. Janson, Poisson Approximation, The Clarendon Press, Oxford University Press, New York, 1992. MR.1163825 (93g:60043)

[4] D.W. Boyd, The spectral radius of averaging operators, Pacific J. Math. 24, no. 1 (1968), 19-28. MR 0221308 (36:4360)

[5] J.A. Fridy, R.A. Goonatilake, and M.K. Khan, On Berry-Esseen bounds of summability transforms, Proc. Amer. Math. Soc. 132, no. 1 (2004), 273-282. MR2021271 (2004j:60047)

[6] F. Galaz and F.J. Solís, Iterating the Cesàro operators, Proc. Amer. Math. Soc. 136, no. 6 (2008), 2147-2153. MR2383520 (2009b:47053)

[7] G.H. Hardy, Divergent Series, Clarendon Press, Oxford, 1949. MR.0030620(11,25a)

[8] I.G. Shevtsova, Sharpening of the upper bound of the absolute constant in the Berry-Esseen inequality, Theory Probab. Appl. 51, no. 3 (2008), 549-553. MR.2325552 (2008f:60025)

Departamento de Métodos Estadísticos, Facultad de Ciencias, Universidad de ZaraGOZA, 50009 ZARAgOZA, SPAIN

E-mail address: adell@unizar.es

Departamento de Métodos Estadísticos, Facultad de Ciencias, Universidad de ZaraGOZA, 50009 Zaragoza, Spain

E-mail address: lekuona@unizar.es 